\title{
Tuning PD and PID Controllers for Double Integrating Plus Time Delay Systems
}

\author{
David Di Ruscio, Christer Dalen
}

Department of Electrical, Information Technology and Cybernetics, University College of Southeast Norway, Porsgrunn, Norway. E-mail: David.Di.Ruscio@usn.no

\begin{abstract}
An existing method for tuning a PI controller for an integrating plus time delay plant are extended to be used for the design of a PD controller for a double integrating plus time delay plant. The PD controller is extended with integral action and an ideal PID controller is suggested in order to achieve optimality of the closed loop responses. Furthermore, some analytical results concerning the proposed PD and PID controller algorithm regarding the relative time-delay margin are worked out and presented. The algorithm and an existing method are successfully compared against each other on some examples, e.g. the planar movement control of a mariner vessel.
\end{abstract}

Keywords: PD and PID controllers, tuning, double integrating system, time delay, maximum time delay error, relative time-delay margin, frequency analysis, robustness, performance.

\section{Introduction}

This paper is concerned with PD and PID controller tuning of Double Integrating Plus Time-Delay (DIPTD) systems or processes. DIPTD processes and close to such processes are common and important processes in, e.g., dynamic positioning systems of ships and other vessels (Schei (1996)). Examples of DIPTD systems may be found in systems where the position/orientation and (angular) velocity are the main states e.g., single axis spacecraft rotation Hughes (1986) and rotary crane motion Gustafsson (1996), in order to mention a few.

In frequency analysis the frequency response $h_{0}(j \omega)$ of the loop transfer function $h_{0}(s)$ is of central importance. The frequency response of the loop transfer function is obtained by putting the Laplace operator $s=j \omega$ where $\omega \geq 0$ is the frequency and $j=\sqrt{-1}$ is the complex number. In a standard feedback system, the loop transfer function $h_{0}(s)$ contains at least the product of the controller transfer function $h_{c}(s)$ and the plant transfer function $h_{p}(s)$. The frequency re- sponse $h_{0}(j \omega)$ is the argument for using the Nyquist and the simplified Bode stability criterion, latter can only be used for stable open loop processes. Notice, that a system is marginally stable if possible poles on the imaginary axis are distinct from one another. However, a DIPTD system is not marginally stable and is defined as an unstable process due to two common poles on the imaginary axis. Notice also that an impulse response of a DIPTD system is unbounded and hence defined unstable.

Different systems may have different controllers. However, often the loop transfer function may be the same or approximately the same. This is a useful observation which we will use to design the PD/PID controller for the DIPTD plant studied in this work. We will be focusing on a classical feedback system with a disturbance $v$, as illustrated in Figure 1.

We will now illustrate that PI controller tuning rules for Integrating Plus Time-Delay (IPTD) plants may be used to design PD controller tuning rules for DIPTD processes, at least when the loop transfer function is the same. Most PI controller tuning rules for IPTD 


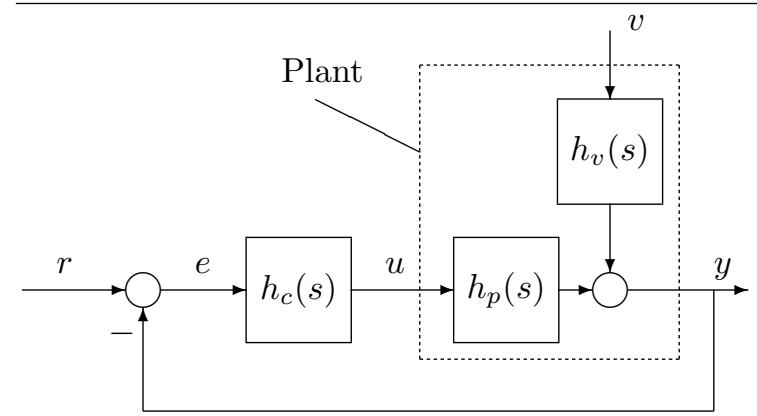

Figure 1: Feedback system. Plant model $h_{p}(s)$, disturbance model $h_{v}(s)$ and controller $h_{c}(s)$. Disturbance $v$ at the input when $h_{v}(s)=h_{p}(s)$ and at the output when $h_{v}(s)=1$.

processes with model

$$
h_{p}(s)=K \frac{e^{-\tau s}}{s}
$$

where $K$ is the gain velocity or the slope of the integrator and, $\tau$, is the time-delay, may be formulated in the following setting Di Ruscio (2010), e.g.

$$
K_{p}=\frac{\alpha}{K \tau}, T_{i}=\beta \tau,
$$

where, $K_{p}$, is the PI controller proportional gain, $T_{i}$, the integral time. Here, $\alpha$, and, $\beta$, are dimensionless parameters, which may be related to each other, e.g. such that $\beta$ is a function of $\alpha$ or vice versa. Hence a PI controller is usually sufficient and of the form

$$
h_{c}(s)=K_{p} \frac{1+T_{i} s}{T_{i} s}
$$

The loop transfer function for a standard feedback systems as in Figure 1 with Eqs. (1) and (3) is

$$
\begin{aligned}
h_{0}(s) & =h_{c}(s) h_{p}(s) \\
& =K_{p} \frac{1+T_{i} s}{T_{i} s} K \frac{e^{-\tau s}}{s} \\
& =\frac{K_{p} K}{T_{i}}\left(1+T_{i} s\right) \frac{e^{-\tau s}}{s^{2}} .
\end{aligned}
$$

Now consider a DIPTD plant $y=h_{p}(s) u$ with model

$$
h_{p}(s)=K \frac{e^{-\tau s}}{s^{2}}
$$

where $K$ is the gain acceleration ${ }^{1}$ and $\tau$ the time-delay. Consider a PD controller of the form

$$
h_{c}(s)=K_{p}\left(1+T_{d} s\right)=K_{p}^{\prime} \frac{1+T_{d} s}{T_{d}},
$$

where we for simplicity of comparison, and using the results in Di Ruscio (2010) Alg. 6.1, have defined the

\footnotetext{
${ }^{1}$ In time domain $K=\frac{\ddot{y}}{u(t-\tau)}$, i.e. gain per square time units.
}

modified proportional gain $K_{p}^{\prime}=K_{p} T_{d}$. It turns out that the loop transfer function of a standard feedback system with a PD controller and a DIPTD model will be of the same form as in Eq. (4), i.e. given by

$$
\begin{aligned}
h_{0}(s) & =h_{c}(s) h_{p}(s) \\
& =K_{p}^{\prime} \frac{1+T_{d} s}{T_{d}} K \frac{e^{-\tau s}}{s^{2}} \\
& =\frac{K_{p}^{\prime} K}{T_{d}}\left(1+T_{d} s\right) \frac{e^{-\tau s}}{s^{2}} .
\end{aligned}
$$

Hence, it turns out that the tuning rules presented in Di Ruscio (2010) may be used to derive PD tuning rules for a DIPTD system. This means that we may deduce PD tuning rules for a DIPTD plant, by replacing $K_{p}$ and $T_{i}$ in the PI tuning algorithm Eq. (2) for the IPTD system with $K_{p} T_{d}$ and $T_{d}$, respectively, in order to derive the PD controller parameters

$$
T_{d}=\beta \tau, K_{p}=\frac{\alpha}{K \tau T_{d}},
$$

for a DIPTD system with model as in Eq. (5).

The PD controller in Eq. (8) is illustrated in Figure 2 (unit reference step response with no disturbances) and Figure 3 (input unit step disturbance with zero reference) with the following parameters, Ziegler and Nichols (1942) where $\alpha=0.71, \beta=3.33$, Tyreus and Luyben (1992) $\alpha=0.42, \beta=7.32$, Skogestad (2003) with $T_{c}=1.5 \tau$ and the method in Di Ruscio (2010) with relative delay margin $\delta=1.6$.

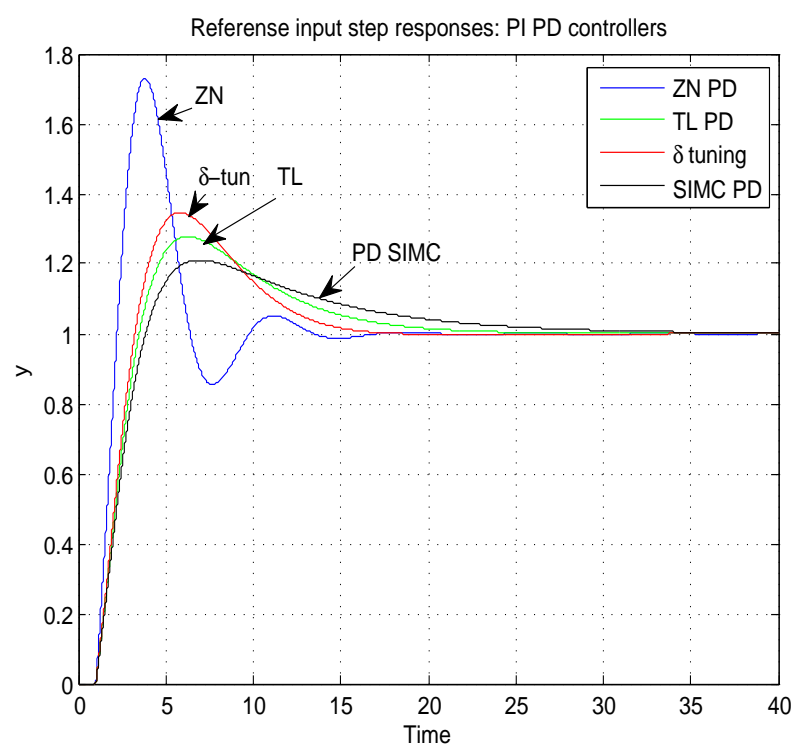

Figure 2: Unit reference step response, no disturbance. PD controllers for a DIPTD plant with $K=$ 1 and $\tau=1$. 


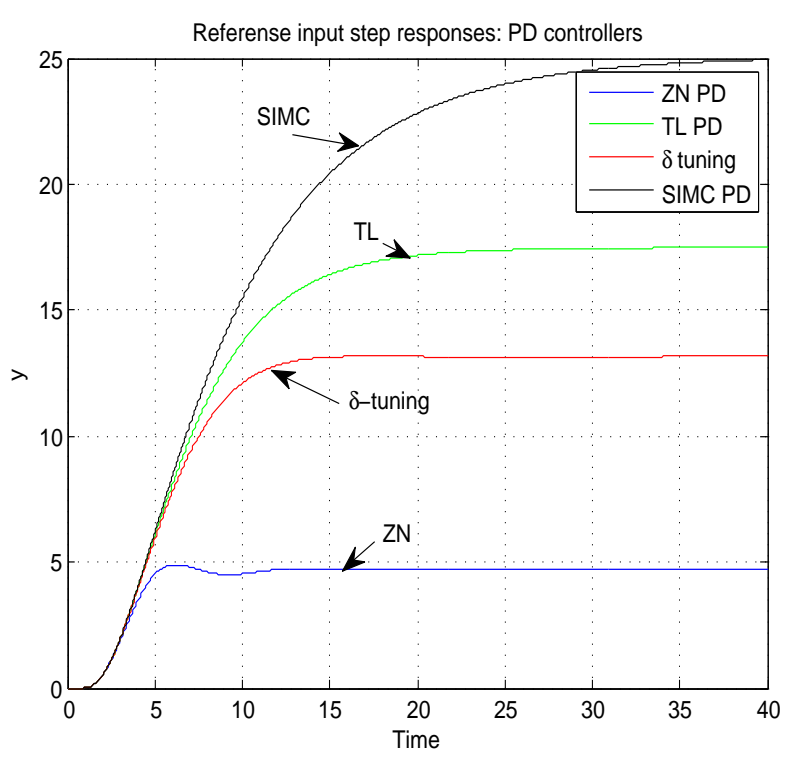

Figure 3: Input unit step disturbance, reference is zero. PD controllers for a DIPTD plant with $K=$ 1 and $\tau=1$.

Theoretically, for a DIPTD process, it is sufficient with a pure Derivative (D) controller. However, due to modeling uncertainties and in case of input disturbances, proportional action is required in order to stabilize the responses and at last a PD controller is needed. In order to eliminate input disturbances, as illustrated in Figure 3, integral action is included and we use a PID controller, in order to ensure the output $y \approx r$ as time goes to infinity.

The main contributions of this paper may be itemized as follows:

- We propose a new algorithm for tuning PD controllers with fixed maximum time-delay error (relative delay margin) for a DIPTD plant in Sec. 2. The algorithm has two tuning parameters, i.e. the relative time-delay margin and a second method constant as tuning parameters.

- We include integral action, and extend the PD controller presented in Sec. 2 and propose an ideal PID controller tuning algorithm with prescribed fixed maximum time-delay error (prescribed timedelay margin) in Sec. 3.

All numerical calculations and plotting facilities are provided by using the MATLAB software, MATLAB (2016). The rest of this paper is organized as follows. In Sec. 2 we propose PD controller tuning rules in terms of a prescribed time-delay margin for a DIPTD plant, and in Sec. 3 we propose an ideal PID controller tuning algorithm for the same plant. Some discussions and definitions are provided in Sec. 4 and simulation examples are presented in Sec. 5. Finally, concluding remarks are given in Sec. 6.

\section{Tuning PD controller with Fixed Relative Time-Delay Margin for DIPTD Plant}

In order to get some insight into the Phase Margin $(P M)$ of the closed loop system and the maximum time-delay error, $d \tau_{\max }$, tolerated before the system becomes unstable, we work out some analytic results in the following, which lead to a new PD controller tuning method for DIPTD processes.

We consider a DIPTD system with model as in Eq. (5) and a PD controller as in Eq. (6). The loop transfer function is given in Eq. (7). The frequency response is given by, $h_{0}(j \omega)=\left|h_{0}(j \omega)\right| e^{j \angle h_{0}(j \omega)}$, where the magnitude is given by

$$
\left|h_{0}(j \omega)\right|=\frac{K_{p} K}{\omega^{2}} \sqrt{1+\left(T_{d} \omega\right)^{2}},
$$

and the phase angle as

$$
\angle h_{0}(j \omega)=-\tau \omega-\pi+\arctan \left(T_{d} \omega\right) .
$$

First, we find the gain crossover frequency, $\omega_{c}$, analytically such that $\left|h_{0}(j \omega)_{c}\right|=1$. From this we find analytic results for the Phase Margin, $P M=\angle h_{0}\left(j \omega_{c}\right)+\pi$, and the maximum time-delay error, $d \tau_{\max }$, such that, $0=P M-d \tau_{\max } \omega_{c}$, in the following. Define a factor, $f$, as

$$
f=\frac{1+\sqrt{1+\frac{4}{\bar{c}^{2}}}}{2}
$$

where the product, $\bar{c}=\alpha \beta$, is constant.

The gain crossover frequency is then given by

$$
\omega_{c}=\sqrt{f} \frac{\alpha}{\tau},
$$

and the PM, in radians,

$$
P M=\delta \sqrt{f} \alpha,
$$

where $\delta$ is defined in the following. The maximum time-delay error may be defined as

$$
d \tau_{\max }=\frac{P M}{\omega_{c}}=\delta \tau,
$$

where we have used Eqs. (12) and (13) in order to derive the last equality in Eq. (14). From this we may define $\delta=\frac{d \tau_{\max }}{\tau}$ as the relative time-delay margin, 
and is the main tuning parameter in the algorithm to be presented. This means that the closed loop system may tolerate a Delay Margin (DM) equal to $d \tau_{\max }=\delta \tau$ where $\delta$ is prescribed by the user. We may express $\delta$ as

$$
\delta=\frac{\arctan (\sqrt{f} \alpha \beta)}{\sqrt{f} \alpha}-1 .
$$

From Eq. (15) we have

$$
\delta=a \frac{1}{\alpha}-1
$$

and with $\bar{c}=\alpha \beta$

$$
\delta=\frac{a}{\bar{c}} \beta-1
$$

where the parameter, $a$, given by

$$
a=\frac{\arctan (\sqrt{f} \bar{c})}{\sqrt{f}},
$$

is a function of $\bar{c}=\alpha \beta$ and constant. Notice that the parameter, $f$, is defined by Eq. (11). We propose the following algorithm for tuning PD controllers for DIPTD plants

\section{Algorithm 2.1 (Max time-delay error tuning) \\ Tuning PD controllers for a DIPTD process. Define} the constant method product parameter

$$
\bar{c}=\alpha \beta \text {. }
$$

From this, we may express, $\beta$, as a linear function of a prescribed time-delay margin $0<\delta$, in order to ensure the stability of the feedback system. We have from Eq. (17)

$$
\beta=\frac{\bar{c}}{a}(\delta+1)
$$

where parameter, a, is defined in Eq. (18). Notice that, $\alpha$, then is found as

$$
\alpha=\frac{\bar{c}}{\beta}=\frac{a}{\delta+1} .
$$

Or equivalently in terms of the PD controller parameters

$$
\begin{gathered}
T_{d}=\beta \tau, \\
K_{p}=\frac{\alpha}{K \tau T_{d}} .
\end{gathered}
$$

The Alg. 2.1 may simply be derived from Alg. 6.1 in Di Ruscio (2010) by replacing $K_{p}$ and $T_{i}$ in that algorithm with $K_{p} T_{d}$ and $T_{d}$, respectively. This is also illustrated in the introduction Sec. 1

The tuning parameter $\bar{c}$ in the PD controller tuning Alg. 2.1 may be chosen relatively wide as

$$
1.5 \leq \bar{c} \leq 4
$$

Notice that the choice $\bar{c}=2.0$ is optimal in the sense that the sensitivity index $M_{s}$ robustness measure is minimized, as illustrated in Figure 4. Performance measured in terms of minimizing Integral Absolute Error (IAE) in case of a reference step response only, is favored by choosing the limit $\bar{c}=4$. The tuning parameter $\bar{c}$ is discussed further in connection with an ideal PID controller tuning in Sec. 3. However, we notice for the moment that the lower limit, $\bar{c}=1.5$ in Eq. (24) may give oscillations in the step and disturbance input/output responses in case of tuning an ideal PID controller.

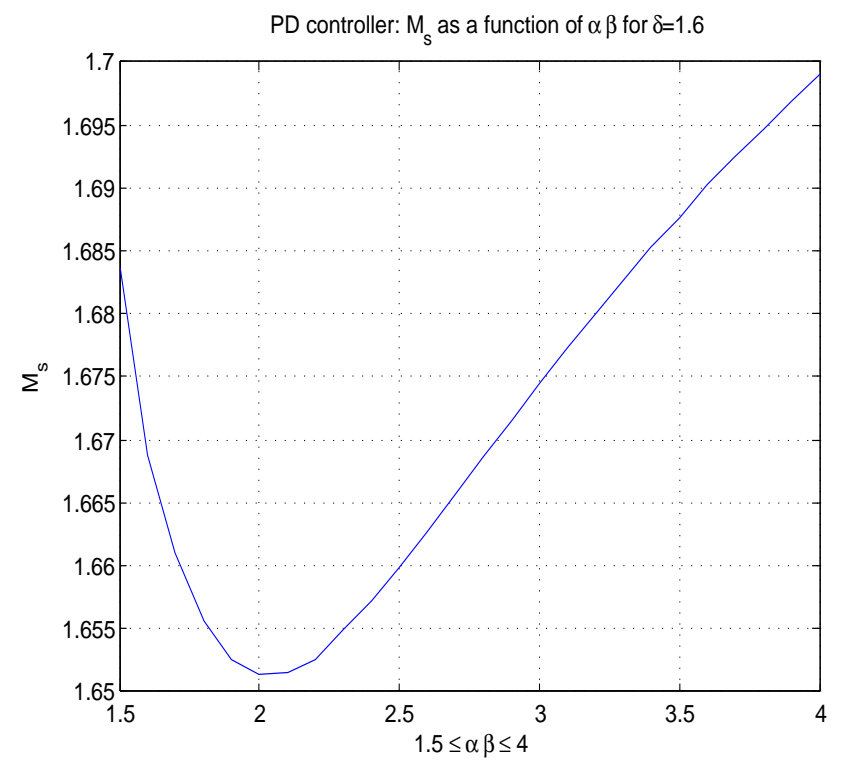

Figure 4: Illustration of sensitivity index $M_{s}$ as a function of the tuning parameter $\bar{c}=\alpha \beta$. PD controller tuning Alg. 2.1 with fixed relative delay error $\delta=1.6$ for a DIPTD plant with $K=1$ and $\tau=1$.

It seems reasonable to choose the relative time-delay error, $0<\delta$, in order to ensure stability. We propose

$$
1.1 \leq \delta \leq 3.5
$$

to archive sufficient robustness. We mention at the end that we have two alternatives for choosing the tuning parameter $\delta$ interpreted as follows.

1. Specify the relative time-delay tuning parameter $0<\delta=\frac{d \tau_{\max }}{\tau}$ in Alg. 2. This means that the 
resulting closed loop system may tolerate a delay margin $D M=d \tau_{\max }=\delta \tau$. Here, in case of a large/small time-delay the DM will be correspondingly larger/smaller resulting in slow/fast responses, and the tuning parameter $\delta$ should be reduced/increased in order to obtain acceptable responses. Margins, $P M, G M$ and $M_{s}$ etc. are constant when varying gain $K \neq 0$ and/or timedelay $0<\tau$ (prescribed $\delta$ and $\bar{c}$ ).

2. If a fixed time-delay margin, independent of $\tau$, is wanted, it makes sense to instead specify $\delta:=\frac{\delta}{\tau}$ as the tuning parameter in Alg. 2.1. This ensures a constant prescribed time-delay margin $D M=$ $d \tau_{\max }=\delta$. This is seen by substituting $\delta$ in Eq. (14) with $\frac{\delta}{\tau}$. The margins $P M, G M, M_{s}$ and $\omega_{c}$ etc. are varying when varying time-delay $0<\tau$ (constant $K \neq 0$ and prescribed $\delta$ and $\bar{c}$ ), but the delay margin $\frac{P M}{\omega_{c}}=\delta$ is constant. Margins are constant when varying gain $K \neq 0$ (constant $0<\tau$ and prescribed $\delta$ and $\bar{c}$ ).

A MATLAB m-file implementation of Alg. 2.1 is provided in App. A. A proof follows the same lines as in Di Ruscio (2010), Sec. 6. The PD tuning Algorithm 2.1 in case of double integrating systems with no timedelay $(\tau \approx 0)$ is discussed in Sec. 4.2.

\section{Tuning PID controller for DIPTD plant}

We propose an ideal PID controller

$$
h_{c}(s)=K_{p}\left(1+\frac{1}{T_{i} s}+T_{d} s\right)
$$

with integral time constant

$$
T_{i}=\gamma T_{d}
$$

where we define $\gamma$ as the relative integral derivative time ratio. Notice that a linear relationship $T_{i}=\gamma T_{d}+$ $\gamma_{0}$ may be justified,as illustrated in Figure 12 and as discussed in Sec. 5.

Here we use the proportional constant $K_{p}$ and the derivative time constant $T_{d}$ as developed in Sec. 2 Alg. 2.1, i.e. Eqs. (23) and (22), respectively. Note that the SIMC, on serial (or cascade) form as described in Skogestad (2004) p. 94, can be written on the ideal form, which corresponds to putting $\gamma=4$ in Eq. (27). Based on numerical experiments we propose

$$
\gamma_{\min } \leq \gamma<\infty
$$

It seems reasonable to chose $1 \leq \gamma_{\min }$. An illustration of the sensitivity index $M_{s}$ as a function of $\bar{c}$ for varying $\gamma$ is illustrated in Figure 8. See also Figure 4 for the limiting case of a PD controller $(\gamma \rightarrow \infty)$ where $M_{s}$ is minimized for $\bar{c}=2$. For decreasing integral derivative ratio $1.6 \geq \gamma \geq \infty$ we find that the minimizing parameter $\bar{c}$ is approximately in the interval $2.1 \leq \bar{c} \leq 2.5$. It seems reasonable to prescribe the method parameter $\bar{c}$ in this interval for given robustness $\delta$ and/or $M_{s}$ and optimize on performance with respect to $\gamma$, e.g. using the IAE for input disturbance as a performance index. As also claimed in Grimholt and Skogestad (2016a) it would be sufficient for DIPTD processes to only consider performance in case of input disturbances. Hence, it makes sense to consider the IAE for input disturbances as a performance index. However, it also makes sense to take output disturbances into consideration and we propose to use as presented in Grimholt and Skogestad (2016a) the Pareto Optimal (PO) criterion $J$ (a function of the IAE for both input and output disturbances) as a function of the sensitivity index $M_{s}$ as a measure for choosing the default tuning parameters $\bar{c}$ and $\gamma$. As illustrated in Figure 5 in order to maximize robustness we chose $\bar{c}=2.5$ and $\gamma=2.1$ as reasonable $\delta$-tuning parameters. Figure 5 also illustrates that the PID tuning algorithm in this paper, Alg. 2.1 and Eq. (27), gives an edge over the SIMC PID tuning rule for a DIPTD process. Remarkably, the margins of using this PID

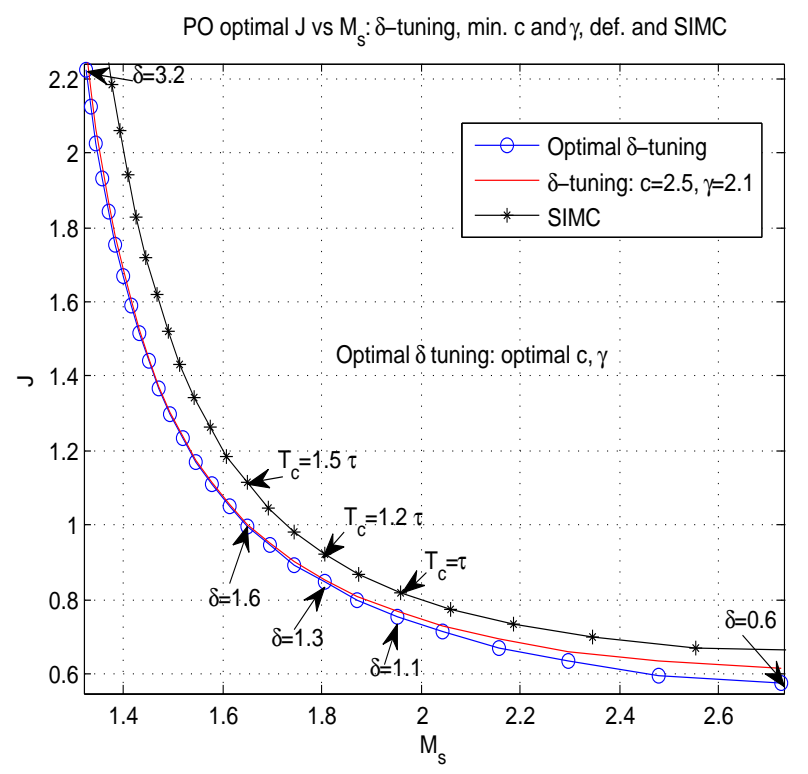

Figure 5: Illustration of performance index $J$ as a function of sensitivity index $M_{s}$. The optimal $\delta$ tuning algorithm with optimal and varying $\bar{c}$ snd $\gamma$ parameters as well as the setting with $\bar{c}=2.5$ and $\gamma=2.1$. SIMC algorithm also illustrated. DIPTD plant with $K=1$ and $\tau=1$. 
controller (Alg. 2.1 and Eq. (27)) on a DIPTD plant have approximately the same margins as using the $\mathrm{PD}$ controller with fixed time-delay margin $\delta$ as developed in Sec. 2, e.g. the normal Gain Margin (GM) is approximately unchanged. See Figure 6 for illustration.

It can be proved that the frequency response of the loop transfer function, in this case, can be expressed as,

$$
h_{0}(j \omega)=\left|h_{0}(j \omega)\right| e^{j \angle h_{0}(j \omega)},
$$

where the magnitude $\left|h_{0}(j \omega)\right|$ and phase angle $\angle h_{0}(j \omega)$ are given as,

$$
\begin{aligned}
\left|h_{0}(j \omega)\right| & =\frac{K_{p} K}{\omega^{2}} \sqrt{1+\left(T_{d} \omega-\frac{1}{\gamma T_{d} \omega}\right)^{2}}, \\
\angle h_{0}(j \omega) & =\arctan \left(T_{d} \omega-\frac{1}{\gamma T_{d} \omega}\right)-\tau \omega-\pi .
\end{aligned}
$$

Note that when $\gamma$ is sufficiently large or $\infty$, we obtain the same results as for the PD controller in Sec. 2, viz. Eqs. (9) and (10). Notice that we may solve Eq. (30) equal to one, for the gain crossover frequency, $\omega_{c}$, analytically but due to the complexity of formulating the solution and that minor advantages of doing so, compared to using Alg. 2.1 and since the margins (normal GM illustrated in Figure 6.) are approximately unchanged, as mentioned above we do not consider this further. However, also notice that the relative timedelay error tuning parameter $\delta$ in the PID tuning algorithm is an approximate relative DM. The difference is marginal and illustrated in Figure 9, where actually we have $0<\delta \leq D M$. Notice that the frequency response Eq. (29) may be used to sketch the Bode- and Nyquist plot, e.g. Figures 6 and 7, respectively. Notice that $G M_{r}$ is the loop gain reduction margin and that we may tolerate a multiplicative loop gain uncertainty, $k_{u}$ in the loop gain, bounded by $G M_{r}<k_{u}<G M$ before instability.

\section{Discussion and Definitions}

\subsection{On the SIMC algorithm}

The SIMC PI controller tuning rules, i.e. the settings for $K_{p}$ and $T_{i}$ in Eq. (3), for an IPTD plant Eq. (1) are given as

$$
K_{p}=\frac{1}{K\left(T_{c}+\tau\right)}, T_{i}=\bar{c}\left(T_{c}+\tau\right),
$$

where $\bar{c}=4$ default in SIMC. However, working with $\bar{c}$ gives more degrees of freedom in the PD/PID algorithm to come. Consider a DIPTD plant as in Eq. (5)

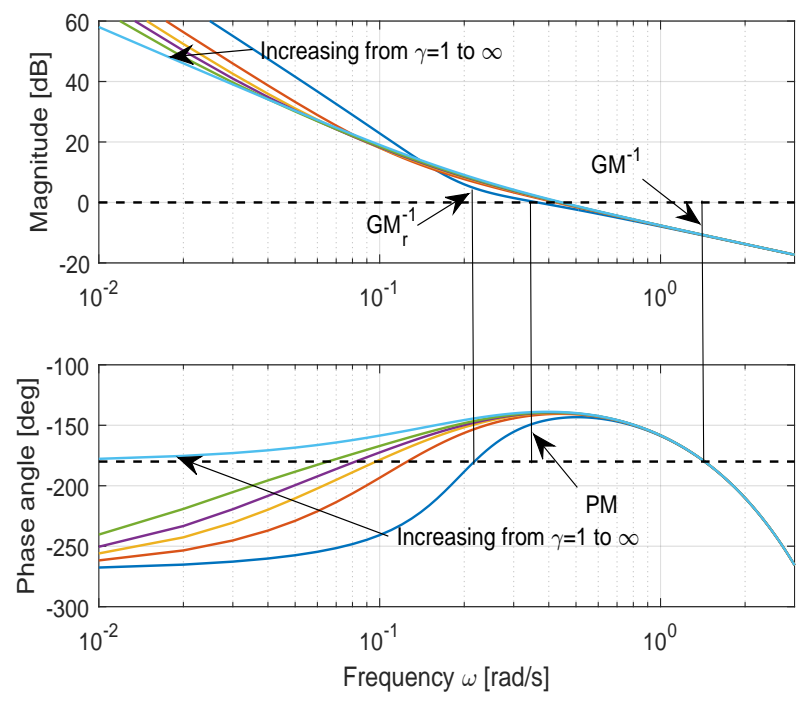

Figure 6: Bode plot of the proposed controller, i.e. Alg. 2.1 and Eq. (27), for a DIPTD plant with $K=1, \tau=1$, fixed $\delta=1.6$ and $\bar{c}=2.1$, and varying $\gamma$ from 1 to $\infty$. $\gamma=\infty$ corresponds to a PD controller and $1 \leq \gamma<\infty$ to PID controllers. Notice that $G M^{-1}$ and $G M_{r}^{-1}$ are not translated to $\mathrm{dB}$ in this plot.

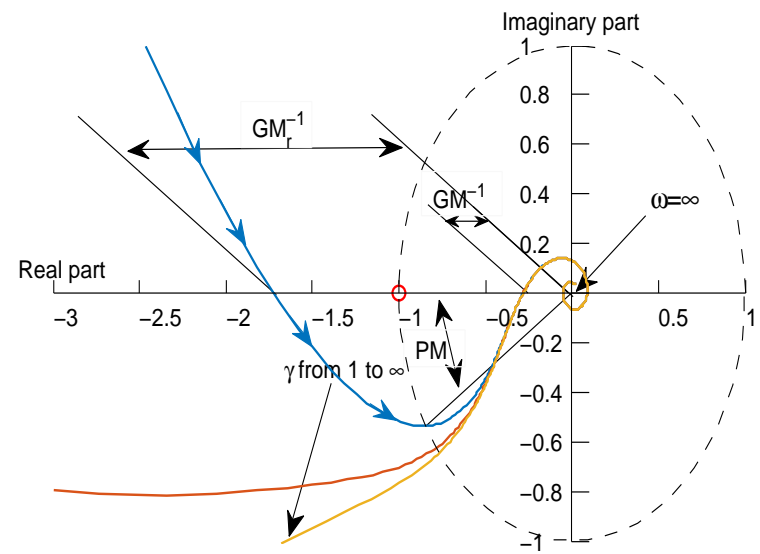

Figure 7: Nyquist plot of the proposed controller, i.e. Alg. 2.1 and Eq. (27), for a DIPTD plant with $K=1$ and $\tau=1$, fixed $\delta=1.6$ and $\bar{c}=2.1$, and varying $\gamma$ from 1 to $\infty \cdot \gamma=\infty$ correspond to a PD controller and $1 \leq \gamma<$ $\infty$ to PID controllers. 


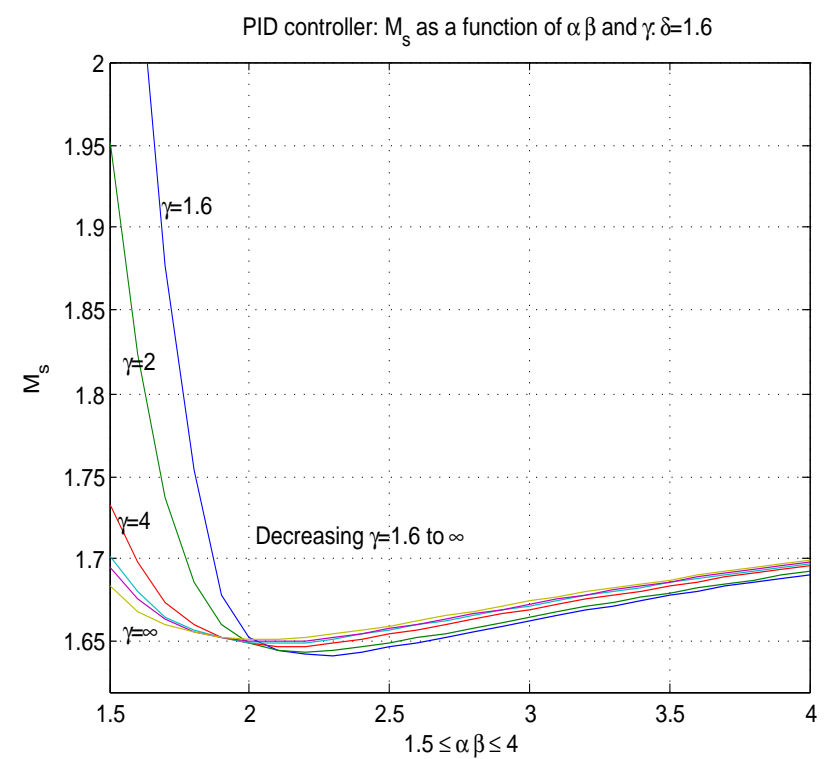

Figure 8: Illustration of sensitivity index $M_{s}$ as a function of the tuning parameter $\bar{c}$ for varying integral derivative ratio $\gamma$. PID controller tuning Alg. 2.1 and Eq. (27) with fixed relative delay margin $\delta=1.6$ for a DIPTD plant with $K=1$ and $\tau=1$.

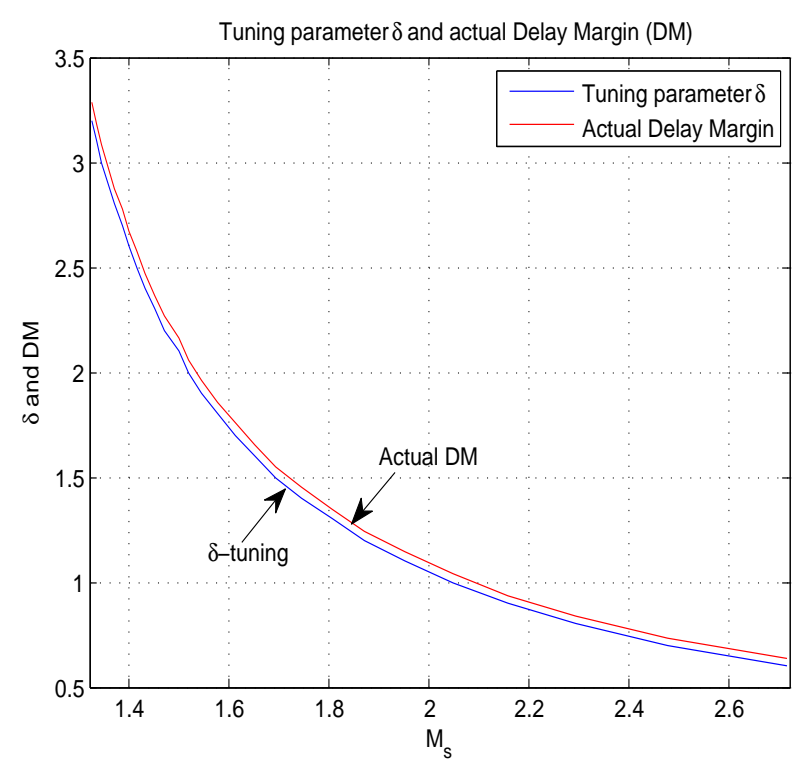

Figure 9: Illustration of the tuning parameter $\delta$ and the actual relative Delay Margin (DM) as a function of robustness $M_{s}$. PID controller tuning Alg. 2.1 and Eq. (27) with varying relative delay margin $\delta$ for a DIPTD plant with $K=1$ and $\tau=1$. with a PD controller as in Eq. (6). Using the same argumentation as in Sec. 1, the loop transfer function Eq. (4) is similar to Eq. (7) if a PD controller as in Eq. (6) is used with setting

$$
\begin{gathered}
T_{d}=\bar{c}\left(T_{c}+\tau\right), \\
K_{p}=\frac{1}{K\left(T_{c}+\tau\right) T_{d}}=\frac{1}{\bar{c} K\left(T_{c}+\tau\right)^{2}},
\end{gathered}
$$

where we have used $T_{d}:=T_{i}$ and $K_{p}:=K_{p} T_{d}$ in the PI tuning rules Eq. (32) in order to derive the PD tuning rules in Eqs. (33) and (34) for a DIPTD plant. This $\mathrm{PD}$ controller tuning may be used on its own.

It is similar to the $\delta$-tuning Alg. 2.1 and notice that the relationship between the tuning parameter $T_{c}$ and the relative time-delay margin tuning parameter $\delta$ is given as presented in Di Ruscio (2010) Eq. (116), i.e.

$$
T_{c}=\left(\frac{\delta+1}{a}-1\right) \tau .
$$

Eq. (35) is obtained by putting $K_{p}$ from Eq. (34) equal to $K_{p}$ in Eq. (23) and using Eq. (21). This PD controller is not considered further.

In SIMC (Grimholt and Skogestad (2016a)) integral action is introduced similarly as a PID controller on cascade/serial form

$$
h_{c}(s)=K_{p} \frac{1+T_{i} s}{T_{i} s}\left(1+T_{d} s\right),
$$

is derived for a 2 nd order process with two large time constants greater than $\bar{c}\left(T_{c}+\tau\right)$ with $\bar{c}=4$. For the DIPTD plant the SIMC PID tuning rules are $K_{p}$ as in (34) and with $T_{i}=T_{d}=\bar{c}\left(T_{c}+\tau\right)$ and the cascade/series PID controller Eq. (36).

Converting the SIMC cascade/serial form Eq. (36), to ideal form as in Eq. (26), we find the corresponding ideal form PID SIMC settings used for comparison purposes,

$K_{p}=\frac{2}{\bar{c} K\left(T_{c}+\tau\right)^{2}}, T_{i}=2 \bar{c}\left(T_{c}+\tau\right), T_{d}=\frac{\bar{c}}{2}\left(T_{c}+\tau\right)$,

and with default SIMC setting $\bar{c}=4$.

\subsection{Tuning PD controller. No time-delay}

In case of double integrating systems with small or no time-delay, i.e. systems with plant models $h_{p}(s)=$ $K \frac{1}{s^{2}}$, the PD tuning algorithm in Alg. 2.1 may still be used. We find from an analysis in the limiting case when $\tau \approx 0$ the PD controller parameters

$$
T_{d}=\frac{\bar{c}}{a} d \tau_{\max }
$$




$$
K_{p}=\frac{a}{K T_{d} d \tau_{\max }}
$$

where the maximum delay error $0 \leq d \tau_{\max }$ is the tuning parameter and parameter $a$ is given in Eq. (18). Furthermore, the gain crossover frequency is in this case given by

$$
\omega_{c}=\frac{\sqrt{f} a}{d \tau_{\max }},
$$

and the PM

$$
P M=\sqrt{f} a .
$$

If integral action is required we propose to include integral action as in Eq. (27) and discussed in Sec. 3, i.e. $T_{i}=\gamma T_{d}$.

\subsection{Performance measures}

In order to quantify performance, we use the Integral Absolute Error $\left(I A E_{x}\right)$ defined as

$$
I A E_{x}=\int_{0}^{\infty}|r-y| d t
$$

where we are using $x:=r$ (reference change, only), $x:=v u$ (input disturbance, only) and $x:=v y$ (output disturbance, only). Furthermore, from Eq. (42) we define:

- $I A E_{r}$ measures the performance in case of a unit step response in the reference, no disturbance.

- $I A E_{v u}$ measures the performance in case of a unit step input disturbance, with the reference equal to zero.

- $I A E_{v y}$ measures the performance in case of a unit step output disturbance, with the reference equal to zero.

See also Figure 1 for more details regarding input and output disturbances.

The amount of input used is evaluated by the Total Value (TV) index formulated in discrete time as

$$
T V=\sum_{k=1}^{\infty}\left|\Delta u_{k}\right|
$$

where, $\Delta u_{k}=u_{k}-u_{k-1}$, is the control rate of change.

The PID controller can be defined optimal in many ways, and in this paper, we mean optimal in the sense that the cost function, as presented in Grimholt and Skogestad (2013), is minimized, which becomes the PO-PID controller.
We consider the following PO criterion

$$
J=s_{r} \frac{I A E_{v y}(p)}{I A E_{v y}^{o}}+\left(1-s_{r}\right) \frac{I A E_{v u}(p)}{I A E_{v u}^{o}},
$$

where $s_{r}$ is the servo-regulator parameter such that $0 \leq s_{r} \leq 1$. Here $s_{r}=0.5$. IAE $E_{v y}^{o}=4.15$ is the reference value based on a PID controller where $M_{s}=1.59$, $I A E_{v u}^{o}=288.56$ is the reference value based on a PID controller where $M_{s}=1.59$ and $p$ is the controller setting, $p=\left(K_{p}, T_{i}, T_{d}\right)$ for the cascade/serial PID controller. Note the definition, $M_{s}=\max _{0 \leq \omega<\infty}\left|\frac{1}{1+h_{0}(s)}\right|$.

Notice further that the origin of this specific value, i.e., $M_{s}=1.59$, is the resulting $M_{s}$-value for a SIMC tuned PI controller with $T_{c}=\tau$ on the process $h_{p}(s)=$ $\frac{e^{-\tau s}}{s+1}$, see Grimholt and Skogestad (2013). The performance index $J$ with $s_{r}=0.5$ in Eq. (44) was originally presented in Grimholt and Skogestad (2012), however Di Ruscio (2012) introduced $0 \leq s_{r} \leq 1$.

\subsection{Default $\delta$-tuning PID parameters $\bar{c}$ and $\gamma$}

The default settings for the parameters $\bar{c}$ and $\gamma$ in the PID $\delta$-tuning Alg. 2.1 and Eq. (27) are justified in the following. Se also Secs. 2 and 3 for background. From the numerical simulation, which is illustrated in Table $1, \bar{c}=1.8$ and $\gamma=2.0$ seems like reasonable choices. In Table 1 the performance criterion $I A E_{v u}$ is calculated from an input step disturbance only. In order to balance performance in terms of both input and output disturbances, we may use the objective function Eq. (44). Taking prescribed robustness $0<\delta$ into account and minimizing the performance index Eq. (44) we obtain numerical results as presented in Tables 2 and 3. The solution to this problem is also illustrated in Figures 5 and 10 and gives the PO $\delta$-tuning $\bar{c}$ and $\gamma$ parameters for specified robustness $\delta$. The presented variables in Tables 1, 3 and Figure 5, is the background for the the setting $\bar{c}=2.5$ and $\gamma=2.1$. It also makes sense to obtain settings for $\bar{c}$ and $\gamma$ by minimizing the MSE given by Eq. (46). Results are shown below in Table 3. See also Table 6 for MSE values from Eq. (46). As also is seen from Table 3 the value of the objective function Eq. (44) for the "default" $\delta$-tuning does not differ very much from the optimal ideal PID controller (optimal with respect to minimizing the performance index Eq. (44) subject to prescribed robustness $M_{s}$ ). This is also illustrated in Figure 5.

In order to obtain simple $\delta$-tuning PID controller settings for the parameters $\bar{c}$ and $\gamma$ we also minimize the MSE over the robust region $1.3 \leq M_{s} \leq 2$ and over the entire region from $1.3 \leq M_{s} \leq 2.7$. These settings as well as an ad hoc chosen choice are presented in Table 4. These settings are further illustrated in the 
Table 1: PID controller, Eq. (26), with the proposed $\delta$-tuning rules in Alg. 2.1 and Eq. (27) for a DIPTD process, $h_{p}(s)=K \frac{e^{-\tau s}}{s^{2}}$, with varying gain, $K$, and time-delay, $\tau$. Shows $\bar{c}_{\text {min }}, \gamma_{\text {min }}=\arg \min _{\bar{c}, \gamma} I A E_{v u}$, with prescribed $\delta=1.6$ (columns 3 and 4 ) and $\delta=3.5$ (columns 5 and 6$)$.

\begin{tabular}{|l|l|l|l|l|l|}
\hline \hline$K$ & $\tau$ & $\bar{c}_{\min }$ & $\gamma_{\min }$ & $\bar{c}_{\min }$ & $\gamma_{\min }$ \\
\hline \hline 1 & 0.1 & 1.6 & 2.1 & 1.8 & 1.8 \\
1 & 0.3 & 2.0 & 1.6 & 1.8 & 1.8 \\
1 & 0.5 & 1.7 & 2.3 & 1.8 & 1.8 \\
1 & 1 & 1.8 & 2.2 & 1.8 & 1.8 \\
1 & 2 & 1.9 & $2-0$ & 1.8 & 2.0 \\
1 & 4 & 1.9 & 2.0 & 1.8 & 2.0 \\
0.1 & 1 & 1.8 & 2.2 & 1.8 & 1.8 \\
0.1 & 2 & 2.0 & 2.0 & 1.8 & 2.0 \\
0.1 & 4 & 1.9 & 2.0 & 1.8 & 2.0 \\
\hline \hline
\end{tabular}

Table 2: PID controller, Eq (26), with the proposed $\delta$ tuning rules in Alg. 2.1 and Eq. (27) for a DIPTD process, $h_{p}(s)=K \frac{e^{-\tau s}}{s^{2}}$, with $K=1$ and time-delay, $\tau=1$. Shows $\bar{c}_{\min }, \gamma_{\min }=$ $\arg \min _{\bar{c}, \gamma} J$, with prescribed $\delta$. Objective in Eq. (44).

\begin{tabular}{|l|l|l|l|l|}
\hline \hline$\delta$ & $\bar{c}_{\min }$ & $\gamma_{\min }$ & $\mathrm{J}$ & $M_{s}$ \\
\hline \hline 1.4 & 2.6 & 2.0817 & 0.9015 & 1.7448 \\
1.5 & 2.5 & 2.0602 & 0.9496 & 1.6932 \\
1.6 & 2.5 & 1.9973 & 0.9998 & 1.6505 \\
1.7 & 2.5 & 1.9939 & 1.0561 & 1.6130 \\
1.8 & 2.4 & 1.9441 & 1.1154 & 1.5769 \\
1.9 & 2.3 & 1.8999 & 1.1760 & 1.5452 \\
2.0 & 2.4 & 1.8701 & 1.2396 & 1.5199 \\
2.1 & 2.5 & 1.8649 & 1.3073 & 1.4979 \\
\hline \hline
\end{tabular}

Table 3: PO-PID parameters. Comparing with optimal $\delta$-tuning and $\delta$-tuning with $\bar{c}=2.5$ and $\gamma=2.1$.

\begin{tabular}{|l|l|l|l|l|l|l|}
\hline \hline & $\delta$ & $K_{p}$ & $T_{i}$ & $T_{d}$ & $M_{s}$ & $\mathrm{~J}$ \\
\hline \hline PID & - & 0.0979 & 11.8081 & 5.0864 & 1.80 & 0.8497 \\
$\delta$-opt & 1.3 & 0.0950 & 11.9674 & 5.3347 & 1.80 & 0.8631 \\
$\delta$-def & 1.3 & 0.0975 & 10.1290 & 5.0645 & 1.80 & 0.8741 \\
\hline PID & - & 0.0787 & 12.5829 & 5.4128 & 1.65 & 0.9988 \\
$\delta$-opt & 1.6 & 0.0763 & 12.9321 & 5.7275 & 1.65 & 1.0018 \\
$\delta$-def & 1.6 & 0.0763 & 12.0227 & 5.725 & 1.65 & 1.0071 \\
\hline PID & - & 0.0305 & 18.3979 & 8.3188 & 1.32 & 2.2277 \\
$\delta$-opt & 3.2 & 0.0305 & 18.7942 & 8.2765 & 1.32 & 2.2518 \\
$\delta$-def & 3.2 & 0.0292 & 19.4213 & 9.2482 & 1.32 & 2.2984 \\
\hline
\end{tabular}

Example Sec. 5. See also Figures 5, 10 and 14 for illustrations.

Table 4: Settings for the $\delta$-tuning PID parameters $\bar{c}$ and $\gamma$ in Alg. 2.1 and Eq. (27). Row 1) optimized over the robust region and 3) over the entire region. See Figures also 5, 10 and 14. Row 2) is an ad hoc chosen choice.

\begin{tabular}{|l|l|l|l|}
\hline \hline $\mathrm{x}$ & $\bar{c}$ & $\gamma$ & Region \\
\hline \hline 1 & 2.24 & 2.24 & $1.3 \leq M_{s} \leq 2.0$ \\
\hline 2 & 2.5 & 2.1 & Ad hoc choice \\
\hline 3 & 2.4 & 2.2 & $1.3 \leq M_{s} \leq 2.7$ \\
\hline \hline
\end{tabular}

\section{Numerical Examples}

The problem of generating the PO-PID curve renders as an optimization problem, i.e.

$$
\min J(p),
$$

subject to $M_{s}(p) \leq M_{s}^{\text {pre }}$.

Here, $J$ is defined as in Eq. (44) and $M_{s}$ is the prescribed value, e.g. chosen in the range $1.3 \leq M_{s} \leq 2.0$ (as suggested in Åström and Hägglund (1995)).

In the incoming examples we solve the optimization problem in Eq. (45) by using the efficient exact gradient algorithm, as presented in Grimholt and Skogestad (2016b). Note that this algorithm was used in Grimholt and Skogestad (2016a).

In order to quantify how close to optimal a given tuning rule is we introduce the Mean Square Error (MSE), as

$$
M S E=\frac{1}{M} \sum_{i=1}^{M}\left(J_{\text {po }}-J_{\text {rule }}\right)^{2},
$$

where $J_{\text {rule }}$ is the curve generated from the given tuning rule, $J_{p o}$ is the PO-PID curve and $M=$ length(1.3: $0.01: 2.0$ ).

In the following examples we are using definitions as POI-PID, POC-PID and POD-PID in order to denote Pareto Optimal Ideal PID controller Eq. (26), Pareto Optimal Cascade PID controller Eq. (36) and Pareto Optimal $\delta$-tuning Alg. 2.1 and Eq. 27, i.o.

\section{Example 5.1 (Reference example)}

In this example we consider the DIPTD process model, $h_{p}(s)=\frac{e^{-s}}{s^{2}}$. We adopt the same reference controllers as used in Grimholt and Skogestad (2016a), viz. the optimal output disturbance controller having $I A E_{v y}=4.15$ and optimal input disturbance controller having $I A E_{v u}=288.56$, given prescribed $M_{s}=1.59$, see Table 6. Note that the optimal input disturbance 
controller does not necessary correspond to the optimal ideal PID controller, since Grimholt and Skogestad (2016a) used the cascade formulation. Notice, that a pure D controller may be more optimal in the sense of output disturbance.

We observe from the performance vs. robustness trade-off curves in Figure 10 that the $\delta$-tuning rule in Algorithm 2.1 and Eq. (27), with setting, $c=\gamma=2.24$ and alternative 1., produces a closer to optimal curve (POI-PID), MSE =0.0002, than the SIMC rule ( $c=$ 4 in Eq. (37)), giving $M S E=0.0584$. Hence, in this case, the $\delta$-tuning rule is $\frac{0.0584}{0.0002}=292$ times more optimal than the SIMC rule (with respect to the corresponding MSE values).

In Figure 11 we see that the optimal DM lies in between the prescribed and exact DM.

It may be beneficial to illustrate a couple of linear approximations based on the POI-PID controllers on the range $1.3 \leq M_{s} \leq 2.0$. Firstly, in Figure 12, we illustrate that the relationship $T_{i}$ vs. $T_{d}$, may be approximated by a first order polynomial, i.e. $T_{i}=$ 2.0437T $T_{d}+1.6216$. Secondly, it turns out that the relation $c_{2}=K K_{p} T_{i} T_{d}$ vs. $M_{s}$, may be approximated by a first order polynomial, i.e. $c_{2}=2.6167 M_{s}+1.2078$, see Figure 13 for illustration.

Table 5: Shows the reference controllers which minimizes the indices $I A E_{v y}$ and $I A E_{v u}$, i.e. optimal output and input disturbance (ideal) PID controllers as in Eq. (36), i.o., given a prescribed $M_{s}=1.59$.

\begin{tabular}{|l|l|l|l|l|l|}
\hline \hline $\mathrm{x}$ & $K_{p}$ & $T_{i}$ & $T_{d}$ & $I A E_{x}^{o}$ & $M_{s}$ \\
\hline \hline$v y$ & 0.02 & $\infty$ & 24.13 & 4.15 & 1.59 \\
\hline$v u$ & 0.07 & 20.24 & 5.06 & 288.56 & 1.59 \\
\hline \hline
\end{tabular}

Table 6: Comparing the trade-off curves, generated from different tuning rules, vs. the POI-PID and POC-PID curves, in terms of MSE in Eq. (46). i.e. the trade-off curves shown in Figure 10.

\begin{tabular}{|l|l|}
\hline \hline Tuning rule & MSE \\
\hline \hline SIMC & 0.0584 \\
\hline POC-PID & 0.0562 \\
\hline$\delta$-tuning $(c=2.5, \gamma=2.1)$ & 0.0015 \\
\hline$\delta$-tuning $(c=2.4, \gamma=2.2)$ & 0.0007 \\
\hline$\delta$-tuning $(c=2.24, \gamma=2.24)$ & 0.0002 \\
\hline $\mathrm{PO} \delta$-PID & $9 \mathrm{e}-06$ \\
\hline \hline
\end{tabular}

Example 5.2 (Critically damped process model) Consider the following process model used in Sec. 6.3
Table 7: POI-PID controllers, with a prescribed $M_{s}$.

\begin{tabular}{|l|l|l|l|l|l|}
\hline \hline$M_{s}$ & $K_{p}$ & $T_{i}$ & $T_{d}$ & $J$ & $D M$ \\
\hline \hline 1.30 & 0.0271 & 19.2724 & 8.7624 & 2.4996 & 3.5034 \\
\hline 1.40 & 0.0416 & 16.3846 & 7.1736 & 1.6625 & 2.6330 \\
\hline 1.59 & 0.0694 & 13.3862 & 5.7675 & 1.0868 & 1.7980 \\
\hline 1.80 & 0.0974 & 11.9603 & 5.0837 & 0.8467 & 1.3380 \\
\hline 2.00 & 0.1215 & 11.2708 & 4.6796 & 0.7305 & 1.0757 \\
\hline \hline
\end{tabular}

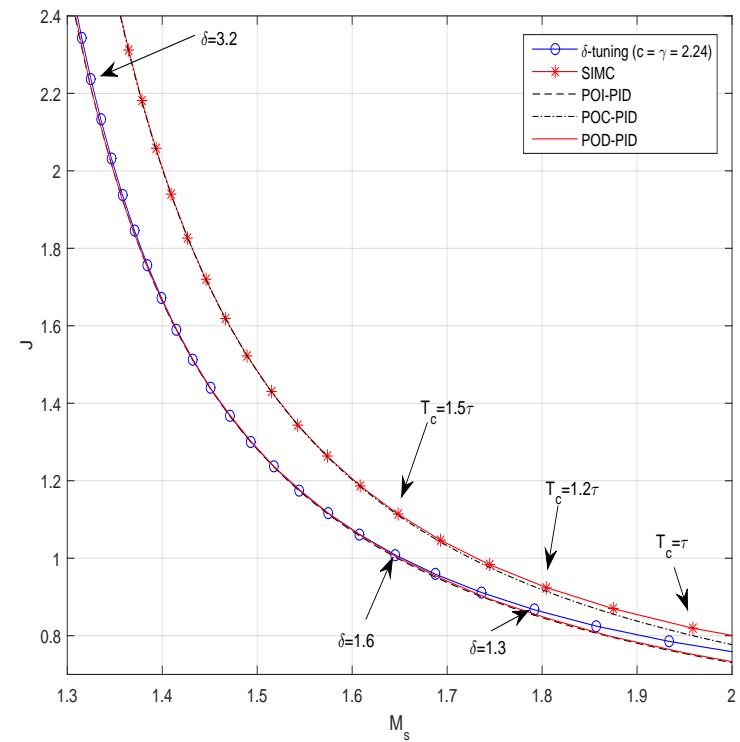

Figure 10: Performance vs. robustness trade-off curves. $I A E_{v y}^{o}=4.15 I A E_{v u}^{o}=288.56$ $s_{r}=0.5$. Comparing the proposed $\delta$-tuning and SIMC vs. the POI-PID controller on the process model, $h_{p}(s)=\frac{e^{-s}}{s^{2}}$. 


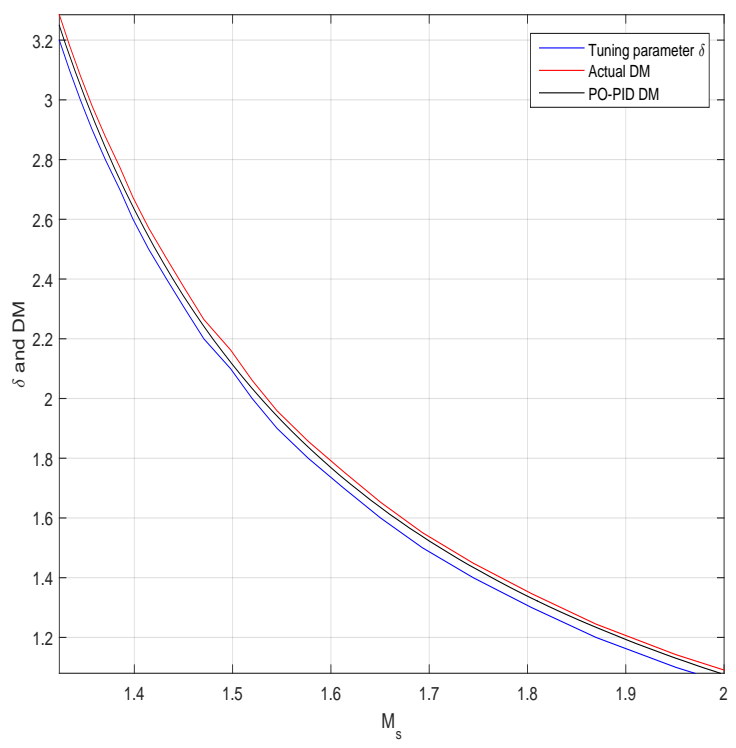

Figure 11: Illustration of the tuning parameter $\delta$, the actual relative Delay Margin (DM) and the POI-PID DM as a function of robustness $M_{s}$. PID controller tuning Alg. 2.1 and Eq. (27) with varying relative delay margin $\delta$ for a DIPTD plant with $K=1$ and $\tau=1$.

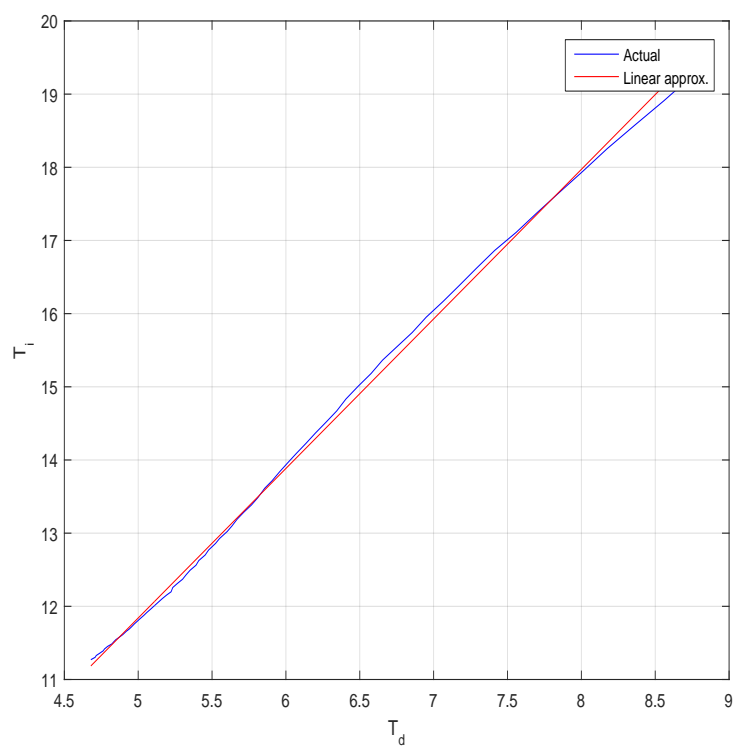

Figure 12: Linear approximation for $T_{i}$ vs. $T_{d}$, for the POI-PID, i.e. $T_{i}=2.0437 T_{d}+1.6216$.

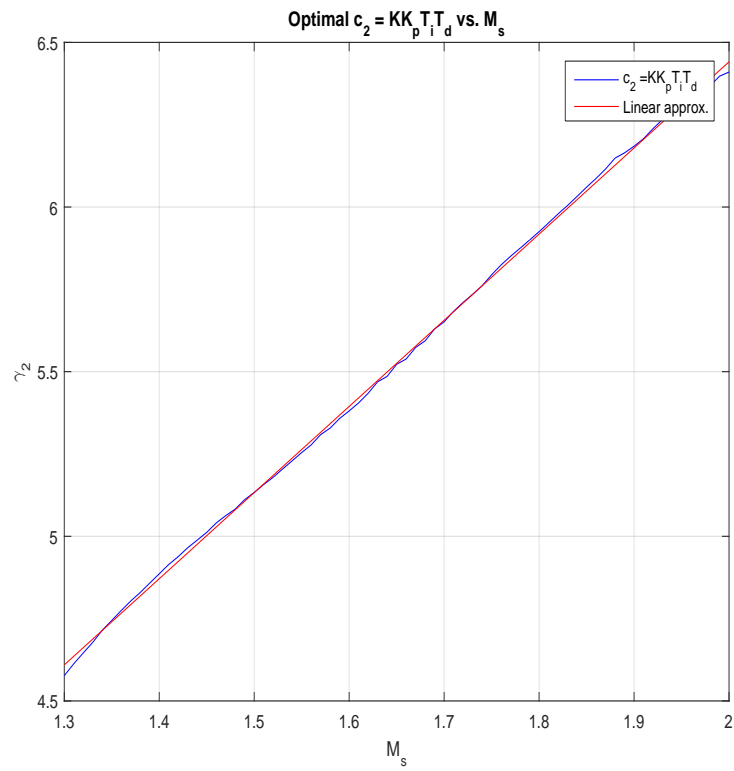

Figure 13: Linear approximation for $c_{2}=K K_{p} T_{i} T_{d}$ vs. $M_{s}$, for the POI-PID, i.e. $c_{2}=2.6167 M_{s}+$ 1.2078 .

in Grimholt and Skogestad (2016a), viz.,

$$
h_{p}(s)=40 \frac{e^{-s}}{(20 s+1)^{2}} .
$$

We may approximate the second order process in Eq. (47) as the following DIPTD process,

$$
h_{p}(s) \approx 0.1 \frac{e^{-s}}{s^{2}} .
$$

We obtain the reference controllers in Table 8. The PO-PID controllers, both ideal and cascade formulation, are generated based on the exact process model given in Eq. (47), while the tuning rules are based on Eq. (48). The results in Figure 14 show poorer performance of the tuning rules, compared to Figure 10 in Example 5.1. Note that, also, in this case, the $\delta$ tuning outperforms the SIMC. Same $\delta$-tuning settings as in Ex. 5.1 are used.

Table 8: Shows the reference controllers which minimizes the indices $I A E_{v y}$ and $I A E_{v u}$, i.e. optimal output and input disturbance (ideal) PID controllers, i.o., given a prescribed $M_{s}=1.59$.

\begin{tabular}{|l|l|l|l|l|l|}
\hline \hline $\mathrm{x}$ & $K_{p}$ & $T_{i}$ & $T_{d}$ & $I A E_{x}^{o}$ & $M_{s}$ \\
\hline \hline $\mathrm{vy}$ & 0.50 & 40.00 & 10.00 & 2.17 & 1.59 \\
\hline $\mathrm{vu}$ & 1.08 & 7.65 & 3.67 & 9.06 & 1.59 \\
\hline \hline
\end{tabular}

\section{Example 5.3 (Vessel example)}

In this example we consider a continuous non-linear 


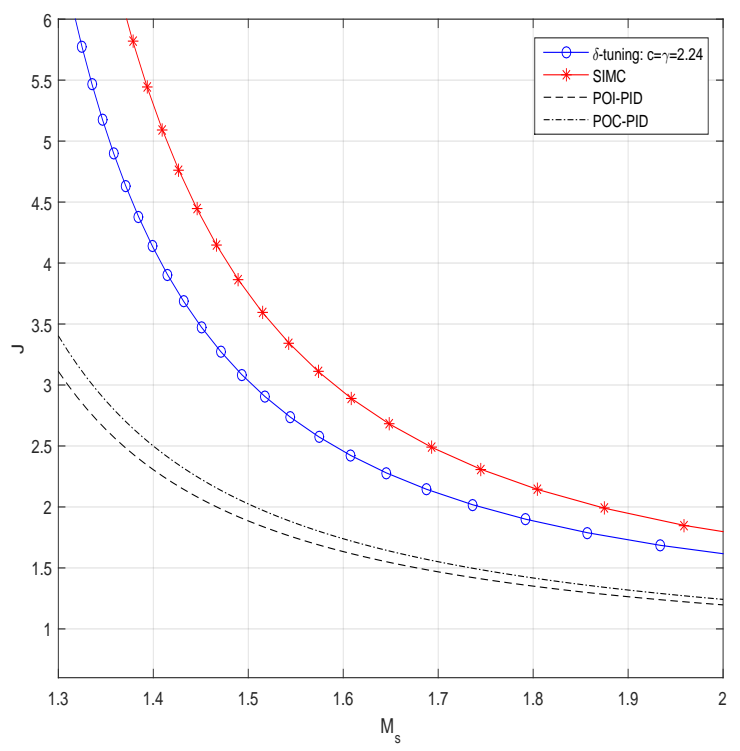

Figure 14: Trade-off curves for performance vs. robustness. $I A E_{v y}^{o}=2.17 I A E_{v u}^{o}=9.06$ Comparing the proposed $\delta$-tuning and SIMC vs. the POI-PID controller on the process model as in Eq. (47).

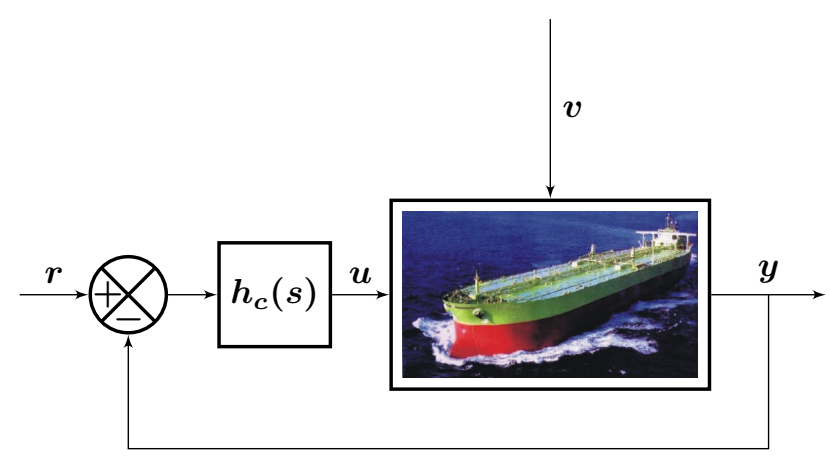

Figure 15: Feedback system. Vessel model and controller $h_{c}(s)$. The input $u$ is the commanded rudder angle and the output $y$ is the perturbed yaw angle about zero. $v$ is disturbance. state space model, implemented in the MSS GNC MAT$L A B$ Toolbox (Fossen and Perez ((2004)), describing the planar movement of a mariner class vessel of length $L=160.93 \mathrm{~m}$, viz.

$$
\dot{x}=f(x, u),
$$

and the measurement equation, as

$$
y=x_{6},
$$

where input, output, and states are defined, i.o., as

$y \in \mathbb{R}:=\{y:$ perturbed yaw angle about zero

$u \in \mathbb{R}:=\{u:$ Commanded rudder angle (rad)

$x \in \mathbb{R}^{7}:=\left\{\begin{array}{l}x_{1}: \text { perturbed surge velocity about Uo }(\mathrm{m} / \mathrm{s}) \\ x_{2}: \text { perturbed sway velocity about zero }(\mathrm{m} / \mathrm{s}) \\ x_{3}: \text { perturbed yaw velocity about zero }(\mathrm{rad} / \mathrm{s}) \\ x_{4}: \text { position in } x \text {-direction }(\mathrm{m}) \\ x_{5}: \text { position in } y \text {-direction }(\mathrm{m}) \\ x_{6}: \text { perturbed yaw angle about zero }(\mathrm{rad}) \\ x_{7}: \text { actual rudder angle }(\mathrm{rad})\end{array}\right.$

See e.g. Skjetne et al. (2004) pp. 4-5 for a physical interpretation of the variables defined above.

We linearize the non-linear model in Eq. (49), numerically, around state $x$ equal to zero-vector and input $u=0$, and obtain the following 7th order model,

$$
h_{p}(s)=\frac{s^{3}}{s^{4}}\left(\frac{0.0037 s+0.0002}{s^{3}+1.1480 s^{2}+0.1503 s+0.0025}\right) .
$$

We show in Figure 16 that the model in Eq. (51) may be approximated by a DIPTD model,

$$
h_{p}(s) \approx 0.0027 \frac{e^{-0.4231}}{s^{2}} .
$$

Using the $\delta$-tuning Alg. 2.1 with $\delta=3.6$ and tune alternative 2., we obtain the following $P D$ controller settings $K_{p}=11.8$ and $T_{d}=8.9$. Figure 17 shows the $P D$ controller implemented on the non-linear model, where we demonstrate a reference step from 0 to $5 \mathrm{deg}$. Note, that a steady state offset, e.g. as in Figure 17, may be counteracted by having a captain in an outer controller loop. But a better idea is to introduce integral action. Note that steady state offsets may be generated from varying disturbances like currents, wave drifting and wind.

The simulation results of the PID controller implementations on the non-linear vessel model are shown in Figures 18, 19 and 20. Here, the vessel is initially headed north until a reference step, $r=5 \mathrm{deg}$, is introduced at time $=20 \mathrm{~s}$. Later on, at time $=150 \mathrm{~s}$, we introduce an input disturbance step, $v=1$. The $\delta$-tuning 


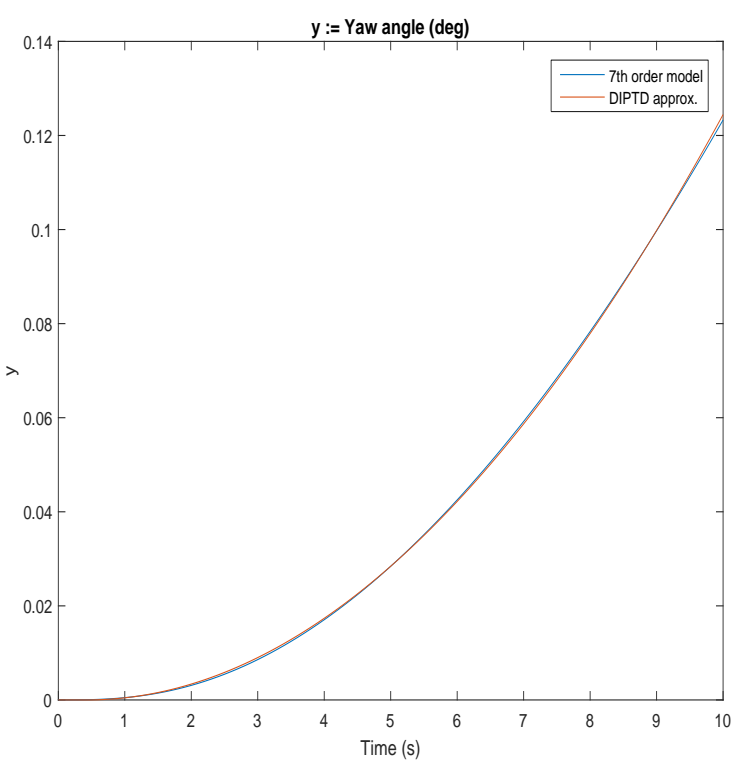

Figure 16: Comparing step responses of the numerically linearized 7 th order model in Eq. (51) vs. the DIPTD process with $K=0.0027$ and $\tau=0.4231$. Shows the optimal DIPTD model, i.e. optimal in the sense that the MSE between the two step responses is minimized.

and the SIMC settings, for prescribed $M_{s}=1.12$, are given in Table 9, where we have used the same settings as for the $P D$ controller above, and $T_{i}=18.6$ from using Eq. (27) with $\gamma=2.1$. Notice, that the SIMC rule gives only better performance in terms of reference step response, i.e. $I A E_{r}=100.7$, however the $\delta$-tuning outperforms the SIMC rule in terms of input disturbance and sum performance, $I A E_{v u}=108.2$ and $I A E=221.4$, i.o., see Table 10. Notice, also that the $T V$ index in Eq. (43) is higher for SIMC, than for the $\delta$-tuning method, see Table 10.

Table 9: Shows $M_{s}$, the controller settings and the DM, for the $\delta$-tuning and the SIMC rule, calculated based on the approximated DIPTD process with $K=0.0027$ and $\tau=0.4231$. The controller settings are used on the non-linear vessel model in Eq. (49). $\delta$-tuning $(c=2.5$ and $\gamma=2.1)$ with $\delta=3.6$. SIMC tuning with $T_{c}=7.9 \tau$.

\begin{tabular}{|l|l|l|l|l|l|}
\hline \hline tun. alg. & $M_{s}$ & $K_{p}$ & $T_{i}$ & $T_{d}$ & $D M$ \\
\hline \hline$\delta(c=2.5, \gamma=2.1)$ & 1.13 & 11.80 & 18.60 & 8.86 & 3.68 \\
\hline$\delta(c=2.4, \gamma=2.2)$ & 1.13 & 12.06 & 18.89 & 8.59 & 3.65 \\
\hline$\delta(c=\gamma=2.24)$ & 1.13 & 12.58 & 18.19 & 8.12 & 3.59 \\
\hline SIMC & 1.13 & 13.06 & 30.12 & 7.53 & 3.52 \\
\hline \hline
\end{tabular}

Table 10: Shows the performance indices $I A E_{r}$, $I A E_{v u}$, sum $I A E$ and $T V$ (Eq. (43)) for the $\delta$-tuning and the SIMC rule, corresponding to the step responses in Figure 18.

\begin{tabular}{|l|l|l|l|l|}
\hline \hline tun. alg. & $I A E_{r}$ & $I A E_{v u}$ & $I A E$ & $T V$ \\
\hline \hline$\delta(c=2.5, \gamma=2.1)$ & 113.26 & 108.17 & 221.43 & 186.20 \\
\hline$\delta(c=2.4, \gamma=2.2)$ & 111.79 & 105.30 & 217.09 & 192.45 \\
\hline$\delta(c=\gamma=2.24)$ & 111.89 & 97.41 & 209.29 & 209.90 \\
\hline SIMC & 100.70 & 132.00 & 232.70 & 198.13 \\
\hline \hline
\end{tabular}
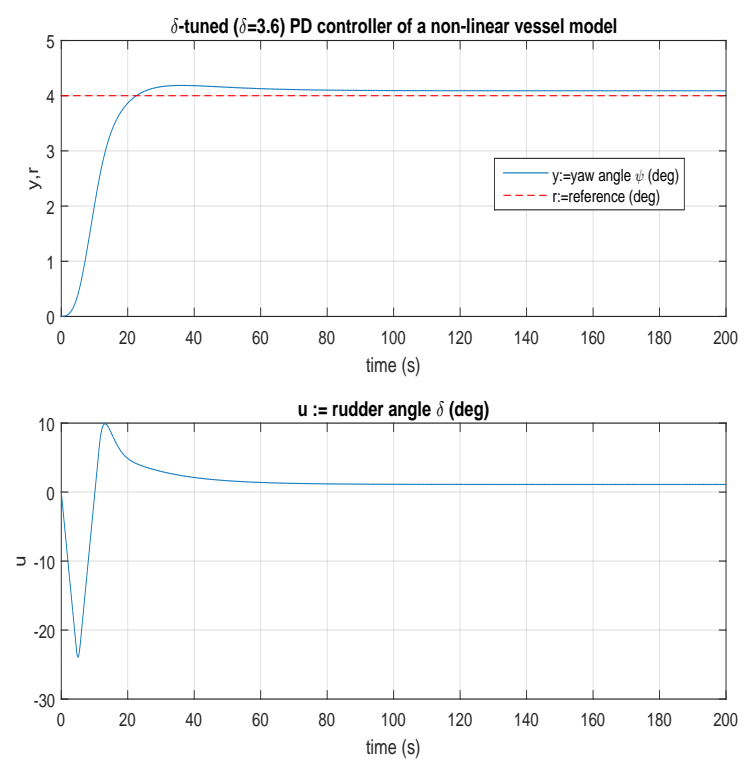

Figure 17: Shows PD control of the non-linear vessel model in Eq. (49). The PD controller is tuned using $\delta$-tuning Alg. 2.1 with prescribed robustness $\delta=3.6$ and tune alternative 2 .

\section{Concluding Remarks}

A new method for tuning PD and PID controllers for a Double Integrating Plus time-delay (DIPTD) process is presented. The proposed PID controller is on ideal form in order to maximize the freedom with respect to optimize on both performance and robustness. The algorithm has in principle only one tuning parameter $0<\delta$ ensuring that the closed loop system has a prescribed delay margin $D M=d \tau_{\max }=\delta \tau$, or alternatively $D M=d \tau_{\max }=\delta$ as an option. Hence, the tuning parameter is the relative delay margin $\delta=\frac{D M}{\tau}$ or optionally the delay margin $D M=\delta$. 

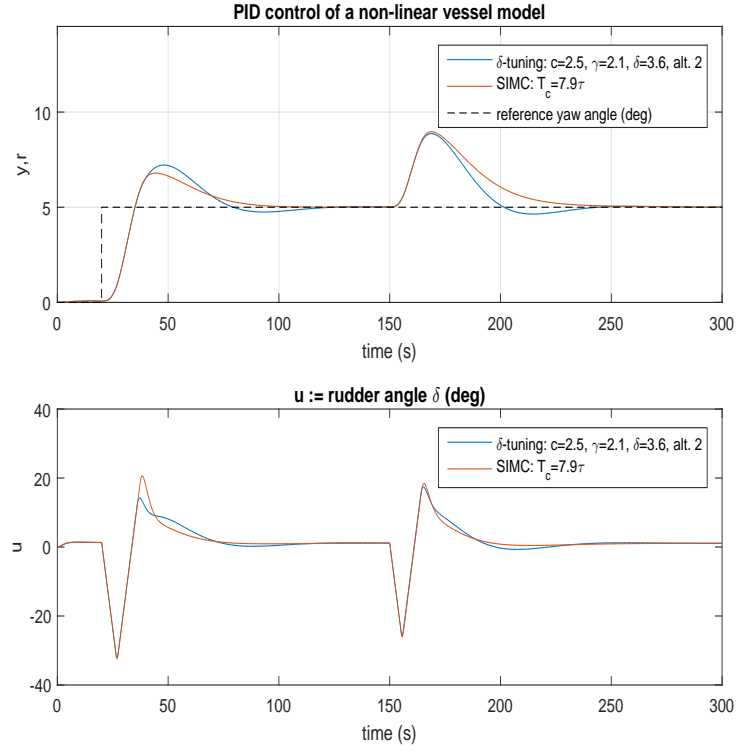

Figure 18: Shows PID control of the non-linear vessel model in Eq. (49). The PID controller is tuned using $\delta$-tuning Alg. 2.1 and Eq. (27), with prescribed robustness $\delta=3.6$ and tune alternative 2 . Reference step, $r=5 \mathrm{deg}$, at time $=20 \mathrm{~s}$. Input disturbance step, $v=1$, is introduced at time $=150 \mathrm{~s}$.

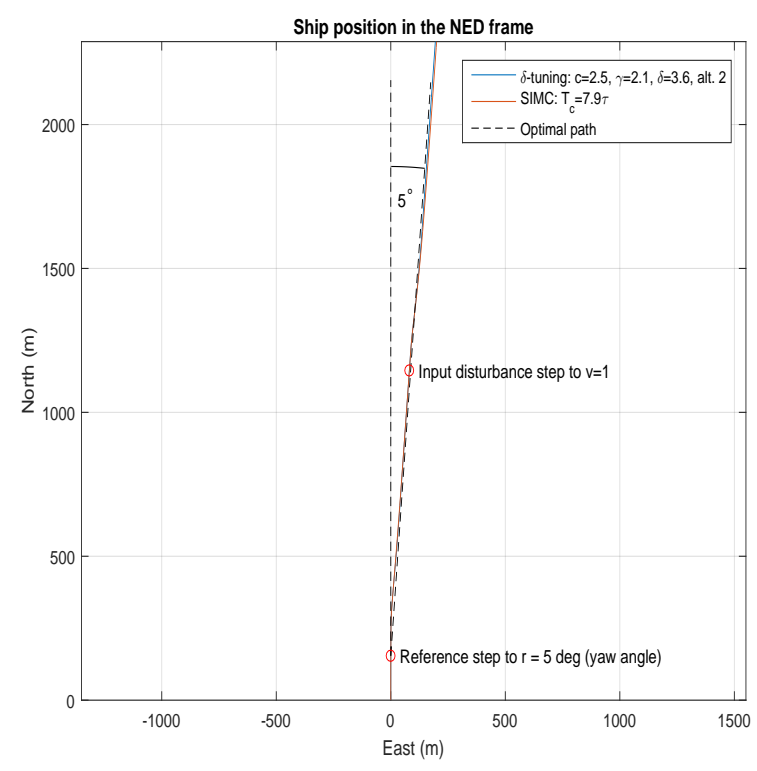

Figure 19: Shows PID control of the non-linear vessel model in Eq. (49). The PID controller is tuned using $\delta$-tuning Alg. 2.1 and Eq. (27), with prescribed robustness $\delta=3.6$ and tune alternative 2 . Reference step, $r=5 \mathrm{deg}$, at time $=20 \mathrm{~s}$. Input disturbance step, $v=1$, is introduced at time $=150 \mathrm{~s}$.

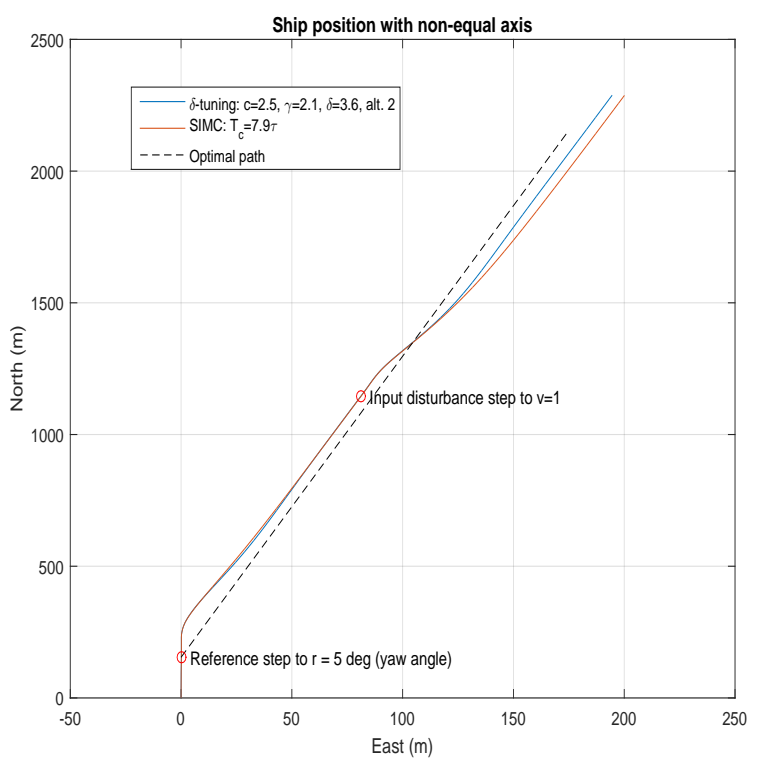

Figure 20: Shows PID control of the non-linear vessel model in Eq. (49). The PID controller is tuned using $\delta$-tuning Alg. 2.1 and Eq. (27), with prescribed robustness $\delta=3.6$ and tune alternative 2 . Reference step, $r=5 \mathrm{deg}$, at time $=20 \mathrm{~s}$. Input disturbance step, $v=1$, is introduced at time $=150 \mathrm{~s}$.

\section{A. MATLAB m-files}

MATLAB m-files for the PD controller tuning Alg. 2.1 and the proposed PID tuning algorithm, for DIPTD plants, are provided in the following. Notice that in the following $\mathrm{m}$-file function pd_tun_maxdelay.m an mfile function pi_tun_maxdelay.m is called. This $\mathrm{m}$-file function is provided in Appendix C in Di Ruscio (2010),

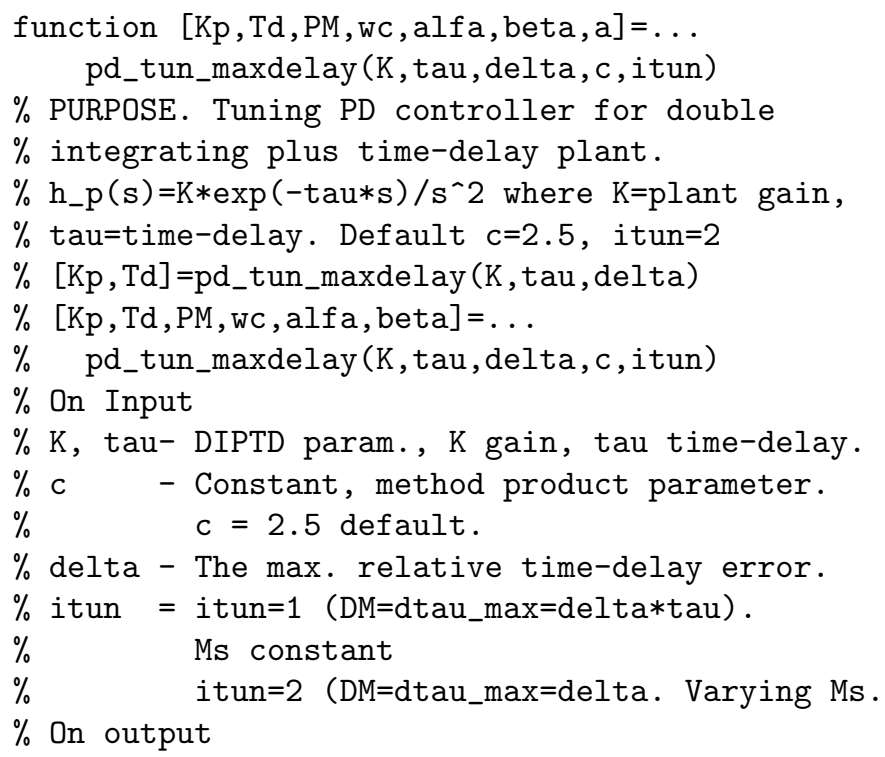




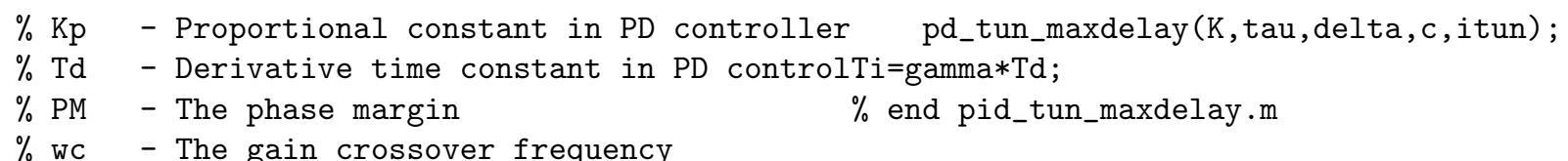

$\%$ alfa - Kp=alfa/ ( $k * t a u)$

$\%$ beta - Ti=beta*tau

$\%$

$\%$ Alg. tuning PD controller for DIPTD process

if nargin $==4$; itun=2; end

if nargin $==3$; itun $=2 ; c=2.5$; end

if nargin $==2$; itun $=2 ; c=2.5 ; \operatorname{delta}=1.6$; end

if itun $==1$

$\%$ delta \% Alg. 2.1 Alt. 1 Alg. 2.1,

$\% \mathrm{DM}=d t a u \_m a x=d e l t a * t a u$. Ms constant. else

delta=delta/tau; \% Alt. 2 Alg. 2.1

$\% \mathrm{DM}=d t a u \_m a x=d e l t a$, varying Ms

end

[alfa, beta ,PM , a,f] =pi_tun_maxdelay (c,delta) ;

$\mathrm{Td}=$ bet $a *$ tau;

$\mathrm{Kp}=\mathrm{alfa} /(\mathrm{K} * \mathrm{tau} * \mathrm{Td}) ; \%$ The PD controller param.

$\mathrm{wc}=\operatorname{sqrt}(\mathrm{f}) * \mathrm{Kp} * \mathrm{~K} * \mathrm{Td}$;

$\%$ end pd_tun_maxdelay.m

function [Kp, Ti, Td, PM, wc, alfa, beta, a]=.. . pid_tun_maxdelay (K, tau,delta, c, gamma, itun)

$\%$ PURPOSE. Tuning PID controller for double

$\%$ integrating plus time-delay plant. Where

$\% \mathrm{~K}=\mathrm{plant}$ gain, tau=time-delay .

$\% \quad[\mathrm{Kp}, \mathrm{Ti}, \mathrm{Td}, \mathrm{PM}, \mathrm{wc}$, alfa, beta, $\mathrm{a}]=\ldots$

$\%$ pid_tun_maxdelay (K,tau,delta)

$\%$ On Input

$\% \mathrm{~K}$, Tau-Model param.

$\%$ delta-The prescribed maximum relative

$\%$ time-delay margin.

$\%$ itun -itun=1 (DM=dtau_max=delta*tau).

$\% \quad$ Ms constant

$\% \quad$ itun=2 (DM=dtau_max=delta.

$\% \quad$ Varying $\mathrm{Ms}$.

$\%$ On output

$\% \mathrm{Kp} \quad$-The proportional constant

$\% \mathrm{Ti} \quad$-The Integral time constant

$\% \mathrm{Td}$-The derivative time constant

$\%$ PM, wc-The phase margin, gain crossover freq.

$\%$ Calls:pd_tun_maxdelay, pi_tun_maxdelay.

$\%$ The algorithm for tuning PID controller for

$\%$ double int+delay process

if nargin $==5$; itun $=2$; end

if nargin $==4$; itun $=2$; gamma $=2.1$; end

if nargin $==3$; itun $=2$; gamma $=2.1 ; c=2.5$; end

if nargin $==2$; itun $=2 ;$ gamma $=2.1 ; c=2.5 ; \ldots$ delta $=1.6 ;$ end

$\% c=2.5 ;$ gamma $=2.1 ; \%$ Default settings

$[\mathrm{Kp}, \mathrm{Td}, \mathrm{PM}, \mathrm{wc}, \mathrm{alfa}, \mathrm{beta}, \mathrm{a}]=$. . .

\section{References}

Åström, K. and Hägglund, T. PID Controllers: Theory, Design, and Tuning. Instrument Society of America, 1995.

Di Ruscio, D. On Tuning PI Controllers for Integrating Plus Time Delay Systems. Modeling, Identification and Control, 2010. 31(4):145-164. doi:10.4173/mic.2010.4.3.

Di Ruscio, D. Pi controller tuning based on integrating plus time delay models: Performance optimal tuning. 2012. In Proceedings of the IASTED Control and Applications Conference. Crete Greece June 18-21.

Fossen, T. I. and Perez, T. Marine systems simulator (mss). (2004). URL http://www.marinecontrol. org.

Grimholt, C. and Skogestad, S. Optimal picontrol and verification of the simc tuning rule. IFAC Proceedings Volumes, 2012. 45(3):11 22. URL http://www.sciencedirect.com/ science/article/pii/S1474667016309934, doi:http://dx.doi.org/10.3182/20120328-3-IT3014.00003 .

Grimholt, C. and Skogestad, S. 11th ifac international symposium on dynamics and control of process systems optimal pid-control on first order plus time delay systems \& verification of the simc rules. IFAC Proceedings Volumes, 2013. 46(32):265 - 270. URL http://www.sciencedirect.com/ science/article/pii/S1474667015382689, doi:http://dx.doi.org/10.3182/20131218-3-IN2045.00122 .

Grimholt, C. and Skogestad, S. Optimal $\{\mathrm{PID}\}$ control of double integrating processes. IFAC-PapersOnLine, 2016a. 49(7):127 - 132. URL http://www.sciencedirect.com/ science/article/pii/S2405896316304359, doi:http://dx.doi.org/10.1016/j.ifacol.2016.07.228. 11th $\{$ IFAC $\}$ Symposium on Dynamics and Control of Process SystemsIncluding Biosystems DYCOPSCAB 2016Trondheim, Norway, 68 June 2016.

Grimholt, C. and Skogestad, S. Optimization of fixedorder controllers using exact gradients. 2016b. URL http://folk.ntnu.no/skoge/publications/ 
2016/grimholt-jpc-pid-exact-gradient/main. pdf. Unpublished.

Gustafsson, T. On the design and implementation of a rotary crane controller. European Journal of Control, 1996. 2(3):166 - 175. URL https://www.diva-portal.org/smash/get/ diva2:988982/FULLTEXT01.pdf.

Hughes, P. Spacecraft attitude dynamics. J. Wiley, 1986.

MATLAB. version 9.1.0.441655 (R2016b). The MathWorks Inc., Natick, Massachusetts,USA, 2016. Control System Toolbox, Version 9.3. Optimization Toolbox, Version 6.2 .

Schei, T. S. Wave disturbance filtering in dynamic positioning systems. Modeling, Identification and Control, 1996. 17(2):87-96. doi:10.4173/mic.1996.2.2.

Skjetne, R., Smogeli, y. N., and Fossen, T. I. A Nonlinear Ship Manoeuvering Model: Identification and adaptive control with experiments for a model ship. Modeling, Identification and Control, 2004. 25(1):327. doi:10.4173/mic.2004.1.1.

Skogestad, S. Simple analytic rules for model reduction and PID controller tuning. Journal of Process Control, 2003. 13(13):291-309. doi:10.1016/S09591524(02)00062-8.

Skogestad, S. Simple analytic rules for model reduction and PID controller tuning. Modeling, Identification and Control, 2004. 25(2):85-120. doi:10.4173/mic.2004.2.2.

Tyreus, B. D. and Luyben, W. L. Tuning PI Controllers for Integrator/Dead Time Processes. Ind. Eng. Chem., 1992. 31(31):2625-2628.

Ziegler, J. and Nichols, N. B. Optimum settings for automatic controllers. Trans. of the A.S.M.E., 1942. 64(64):759-768. 